Supporting Information

\title{
Highly Microporous Activated Carbons with Industrial Potential for Selective Adsorption of Ethane over Ethylene
}

Zhonghao Wang ${ }^{\dagger}$, Lifeng Yang ${ }^{{ }^{*}}$, Peixin Zhang ${ }^{\dagger}$, Jiyu Cui ${ }^{\dagger}$, Peikun Chen ${ }^{\dagger}$, Qi Ding ${ }^{\dagger}$, Xili Cuit, Huabin Xing ${ }^{\dagger, t^{*}}$

$\dagger$ Key Laboratory of Biomass Chemical Engineering of Ministry of Education, College of Chemical and Biological Engineering, Zhejiang University, Hangzhou 310027, China.

$\ddagger$ Hangzhou Global Scientific and Technological Innovation Center, Zhejiang University, Hangzhou 311215, China.

*Corresponding authors. Email: lifeng_yang@zju.edu.cn, xinghb@zju.edu.cn 


\section{Experimental Part}
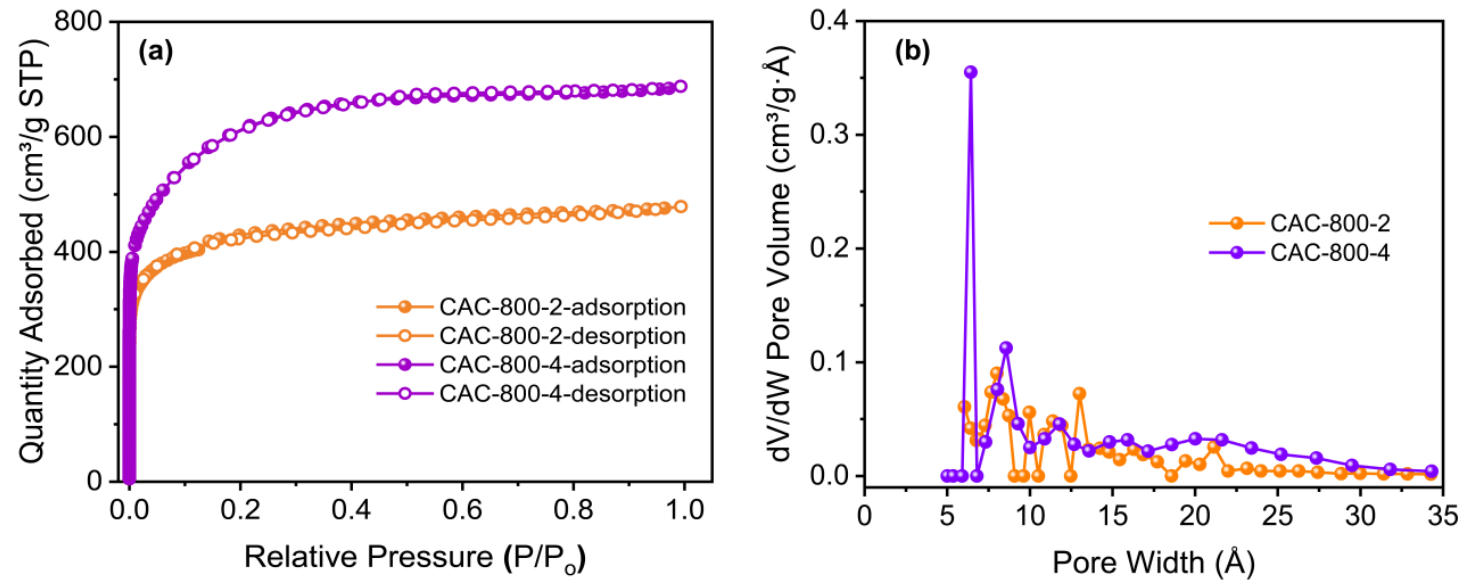

Figure S1. (a) $\mathrm{N}_{2}$ adsorption-desorption isotherms of CAC-800-X at $77 \mathrm{~K}$; (b) DFT calculated pore-size distribution of CAC-800-X;

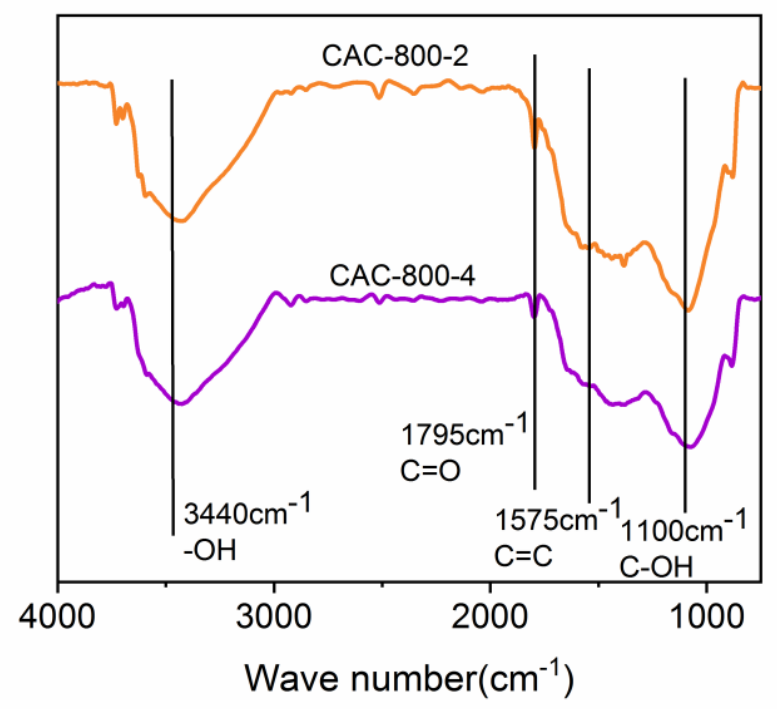

Figure S2. The FTIR spectra of CAC-800-2 and CAC-800-4. 

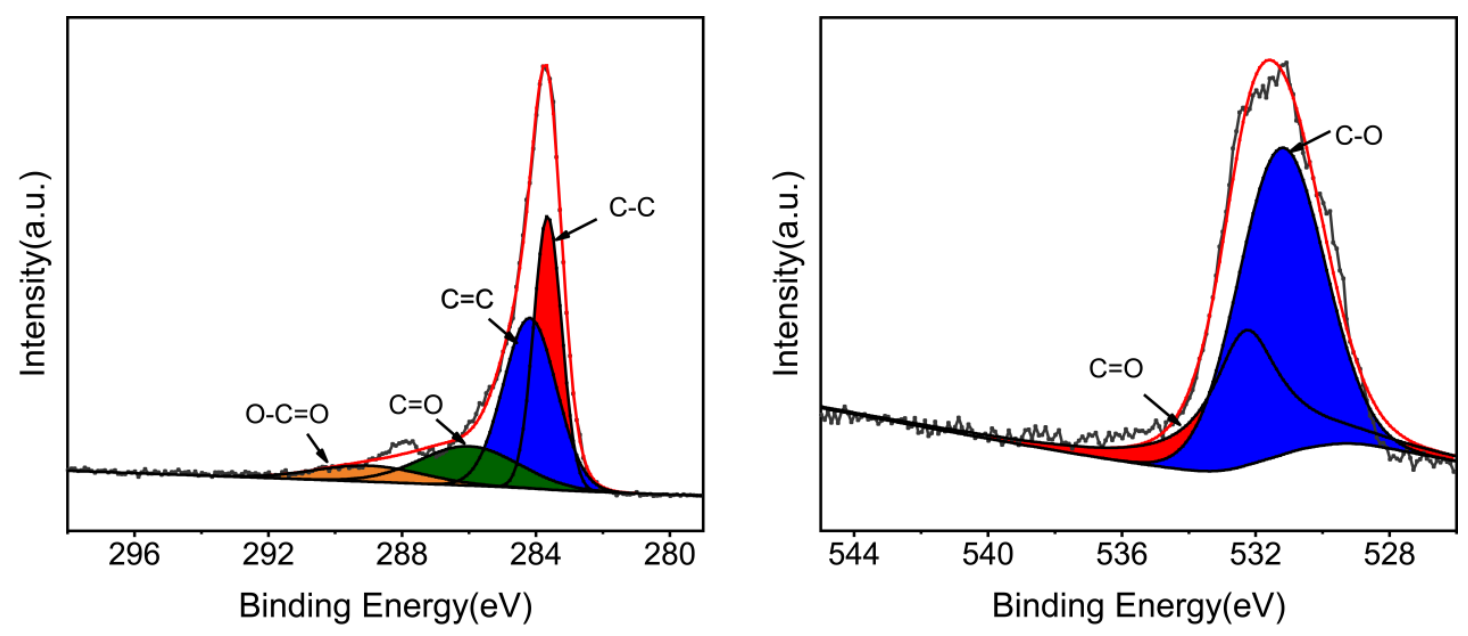

Figure S3. (a) C1s XPS spectrum of CAC; (b) O1s XPS spectrum of CAC.
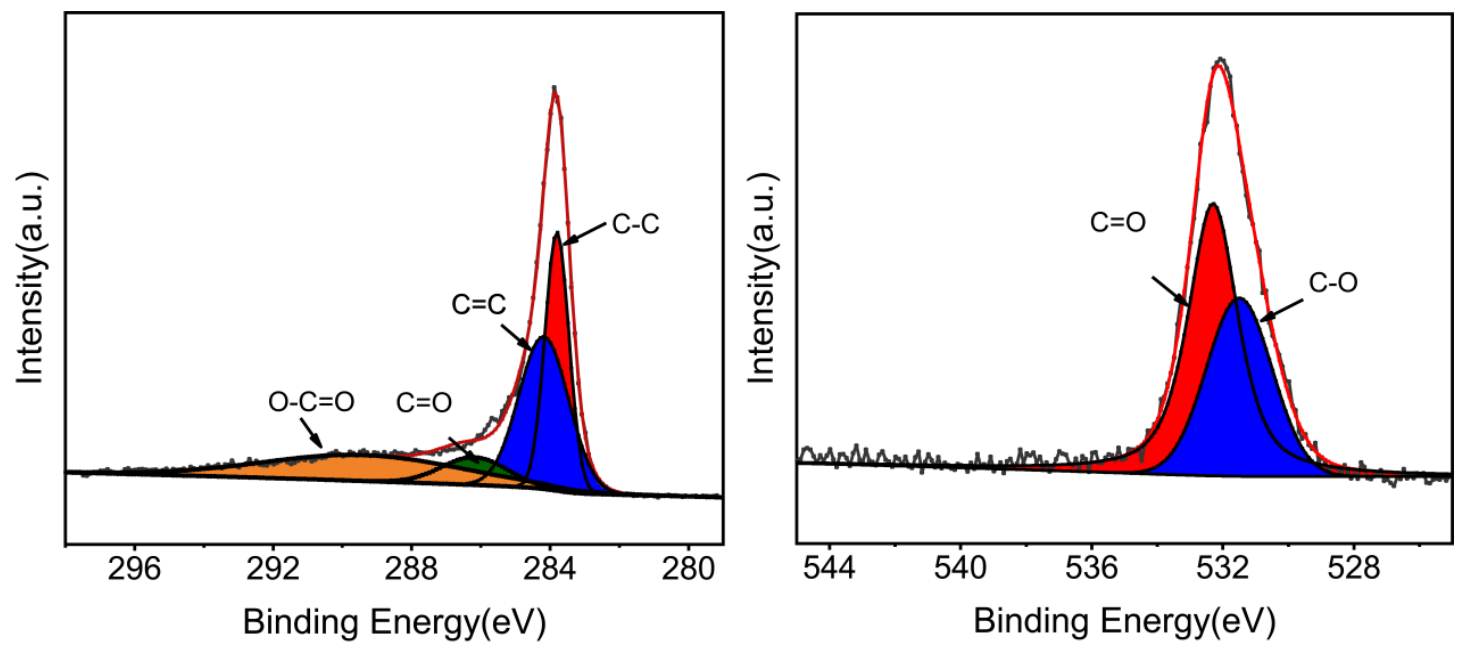

Figure S4. (a) C1s XPS spectrum of CAC-700-3; (b) O1s XPS spectrum of CAC-700-3. 

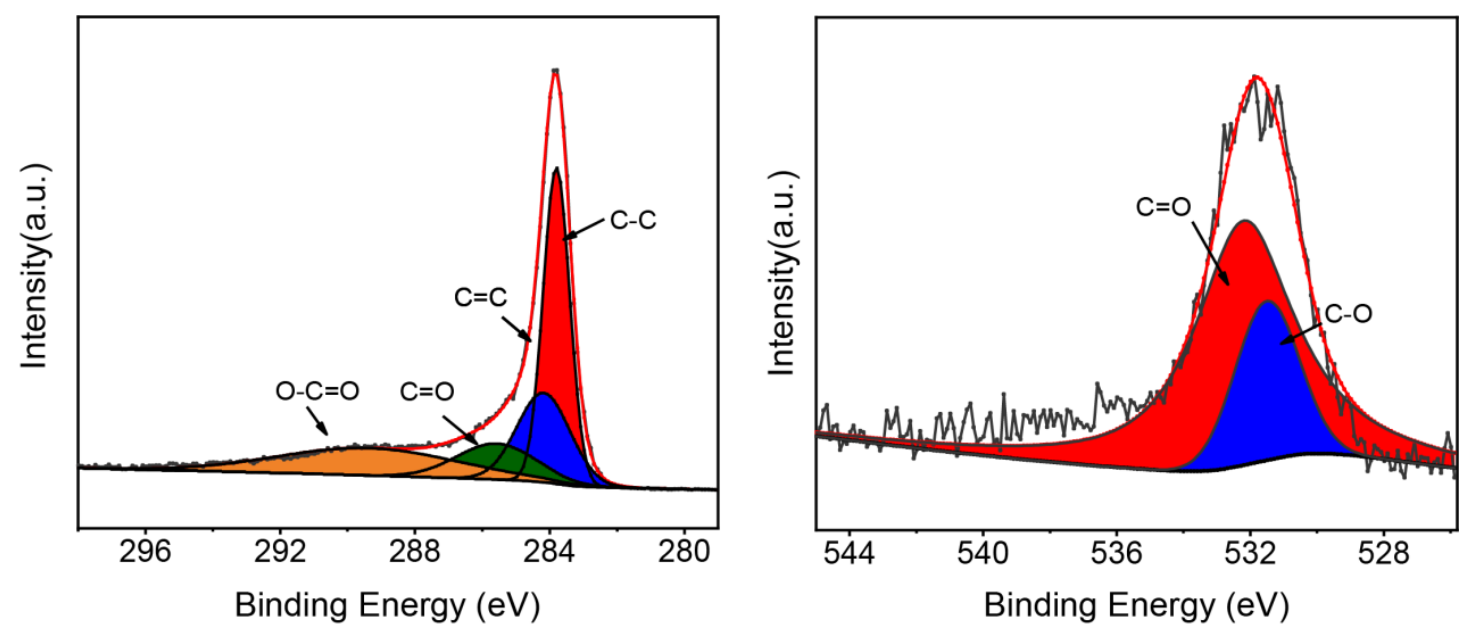

Figure S5. (a) C1s XPS spectrum of CAC-900-3; (b) O1s XPS spectrum of CAC-900-3.
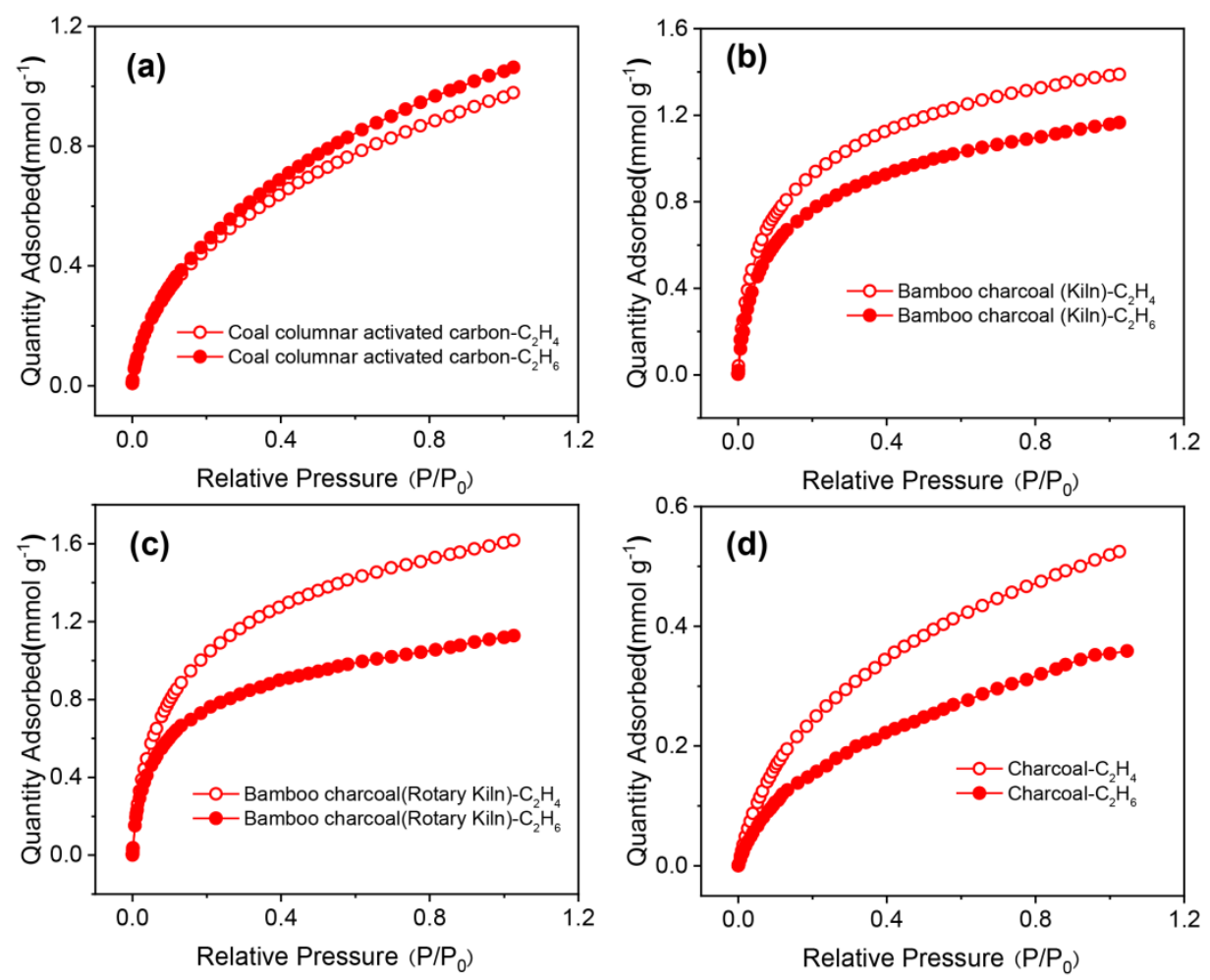

Figure S6. $\mathrm{C}_{2} \mathrm{H}_{4} / \mathrm{C}_{2} \mathrm{H}_{6}$ adsorption isotherms at $298 \mathrm{~K}$ of (a) Coal columnar activated carbon; (b)Bamboo charcoal (Kiln); (c) Bamboo charcoal (Rotary Kiln); (d) Charcoal.. 


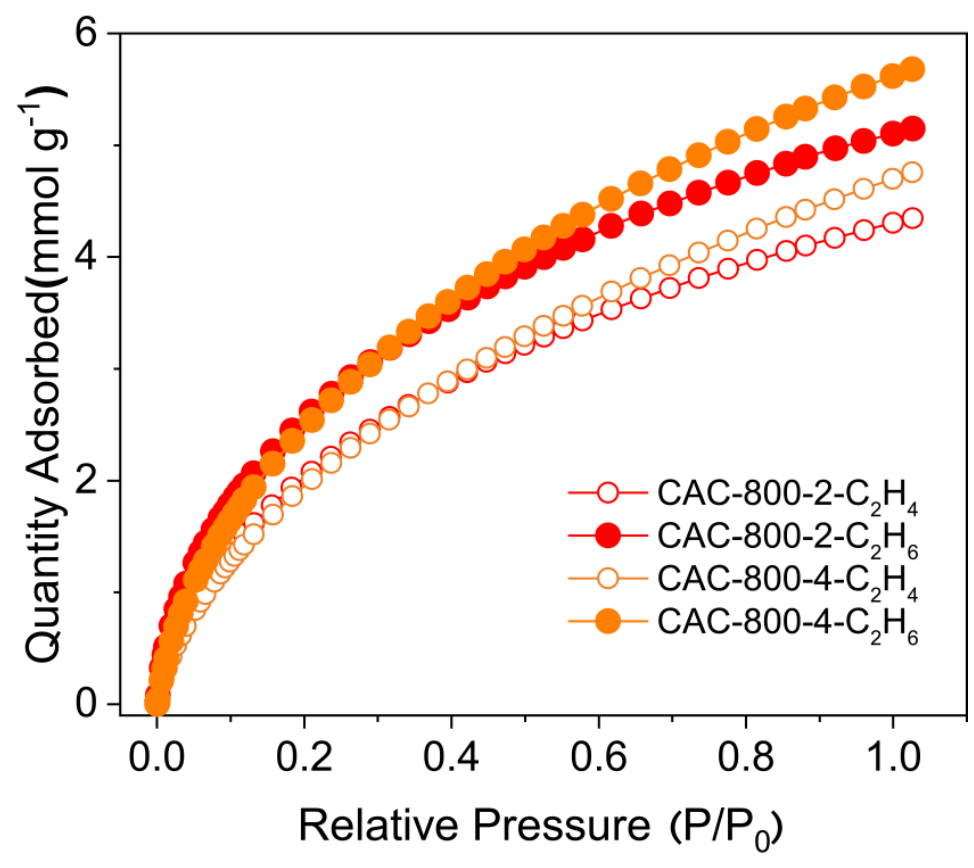

Figure S7. $\mathrm{C}_{2} \mathrm{H}_{4}$ and $\mathrm{C}_{2} \mathrm{H}_{6}$ adsorption isotherms of CAC-800-2 and CAC-800-4 at $298 \mathrm{~K}$. 

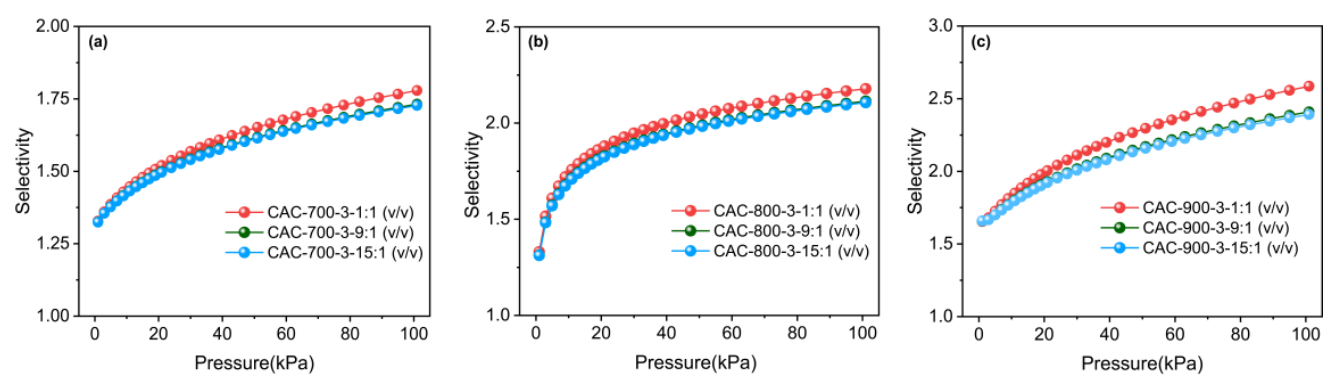

Figure S8. IAST-predicted selectivity for different $\mathrm{C}_{2} \mathrm{H}_{4} / \mathrm{C}_{2} \mathrm{H}_{6}$ mixtures $(1: 1,9: 1$, 15:1 v/v) at $298 \mathrm{~K}$ on (a) CAC-700-3; (b) CAC-800-3; (c) CAC-900-3.
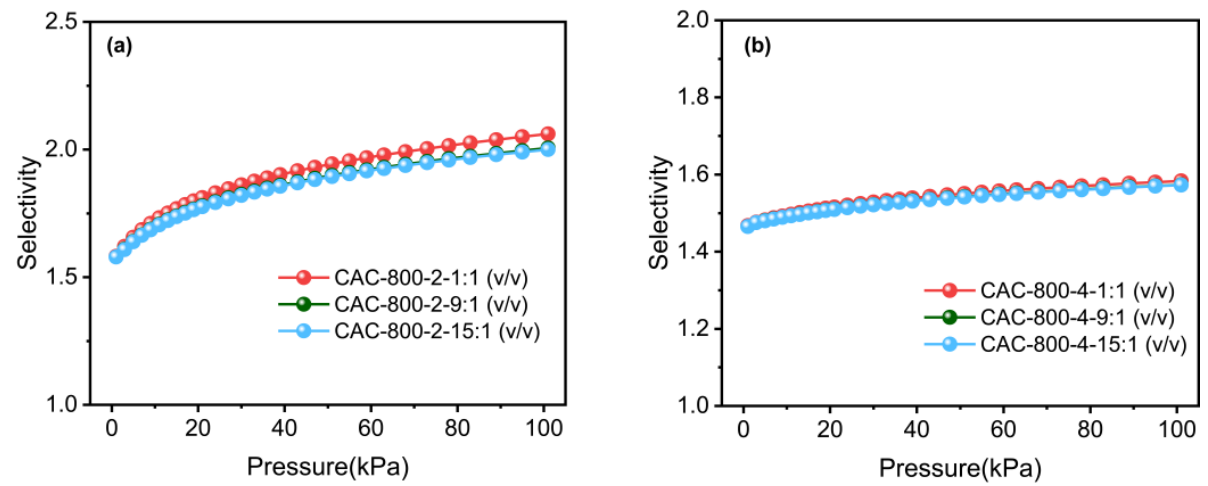

Figure S9. IAST-predicted selectivity for different $\mathrm{C}_{2} \mathrm{H}_{4} / \mathrm{C}_{2} \mathrm{H}_{6}$ mixtures $(1: 1,9: 1$, $15: 1 \mathrm{v} / \mathrm{v}$ ) at $298 \mathrm{~K}$ on (a) CAC-800-2; (b) CAC-800-4. 

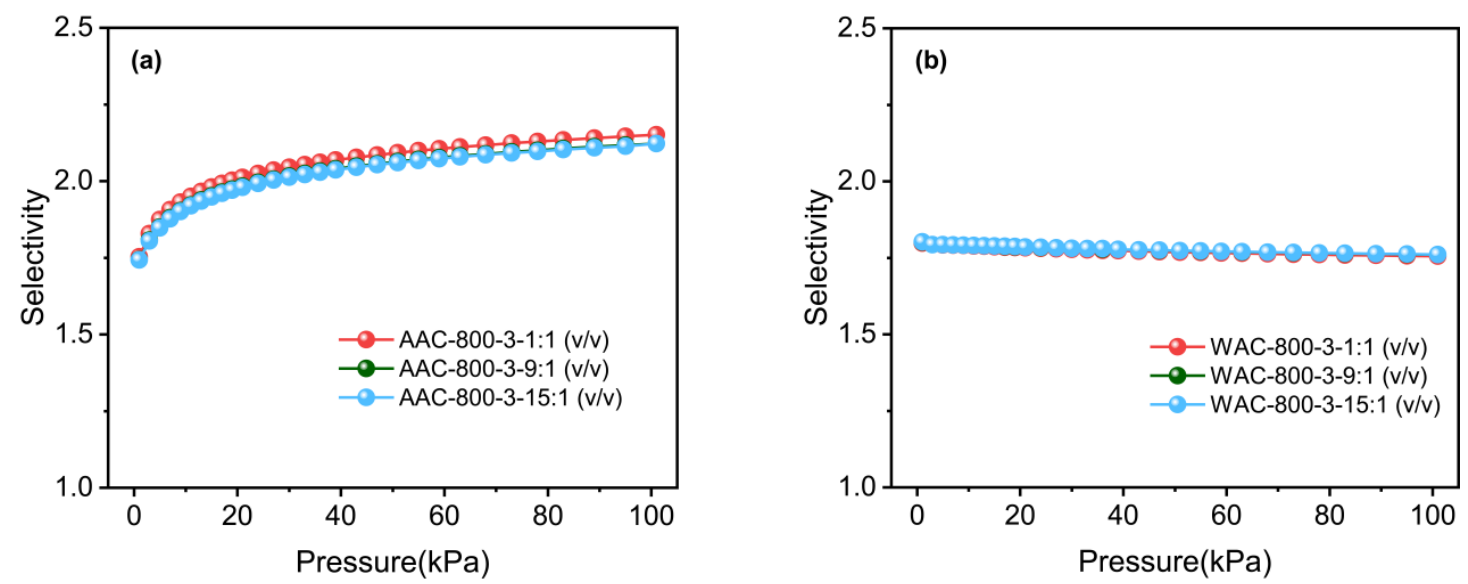

Figure S10. IAST-predicted selectivity for different $\mathrm{C}_{2} \mathrm{H}_{4} / \mathrm{C}_{2} \mathrm{H}_{6}$ mixtures (1:1, 9:1, 15:1 v/v) at $298 \mathrm{~K}$ on (a) AAC-800-3; (b) WAC-800-3.
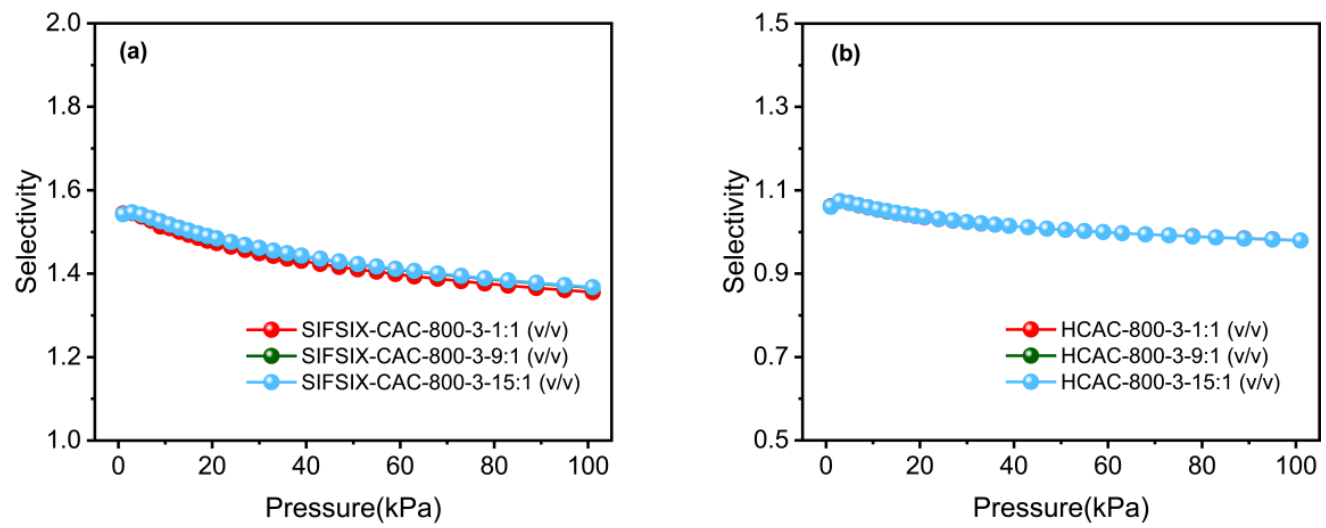

Figure S11. IAST-predicted selectivity for different $\mathrm{C}_{2} \mathrm{H}_{4} / \mathrm{C}_{2} \mathrm{H}_{6}$ mixtures (1:1, 9:1, 15:1 v/v) at $298 \mathrm{~K}$ on (a) SIFSIX-CAC-800-3; (b) HCAC-800-3. 

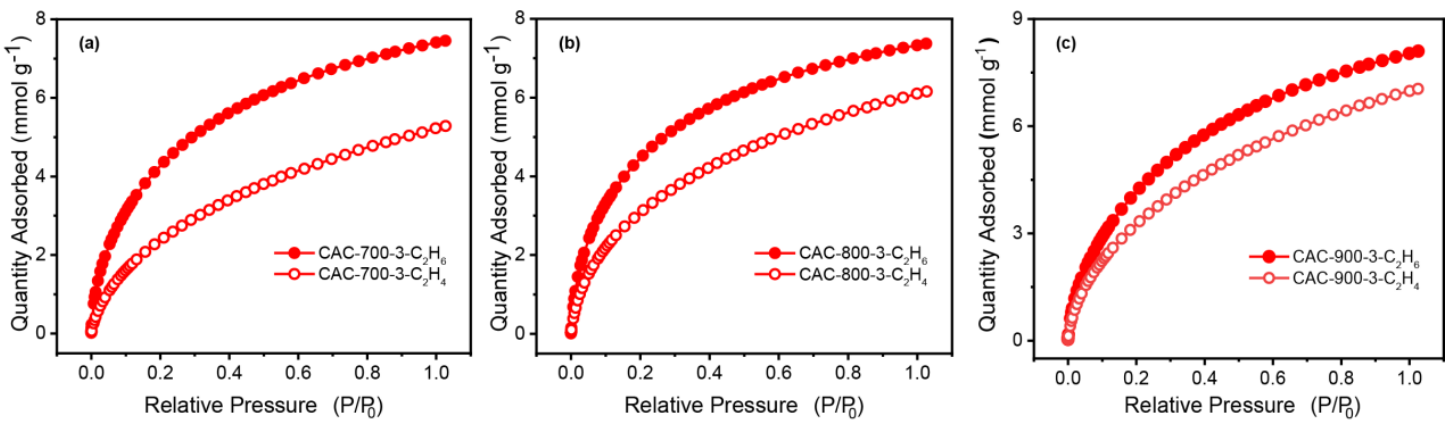

Figure S12. $\mathrm{C}_{2} \mathrm{H}_{4}$ and $\mathrm{C}_{2} \mathrm{H}_{6}$ adsorption isotherms at $273 \mathrm{~K}$ of (a) CAC-700-3; (b) CAC-800-3; (b) CAC-900-3.
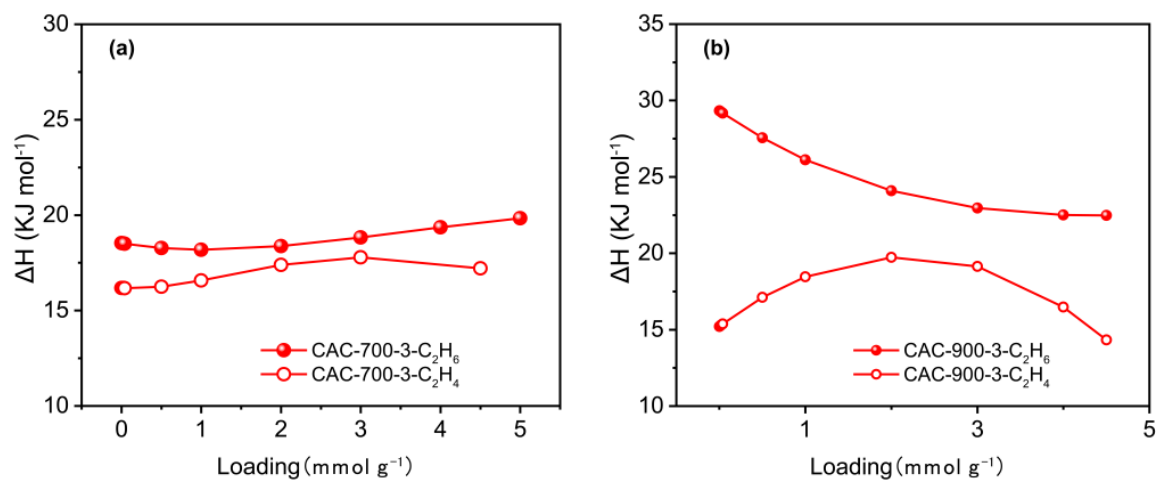

Figure S13. (a) The isosteric heat of $\mathrm{C}_{2} \mathrm{H}_{4}$ and $\mathrm{C}_{2} \mathrm{H}_{6}$ adsorption over CAC-700-3;

(b) The isosteric heats of $\mathrm{C}_{2} \mathrm{H}_{4}$ and $\mathrm{C}_{2} \mathrm{H}_{6}$ adsorption over CAC-900-3. 


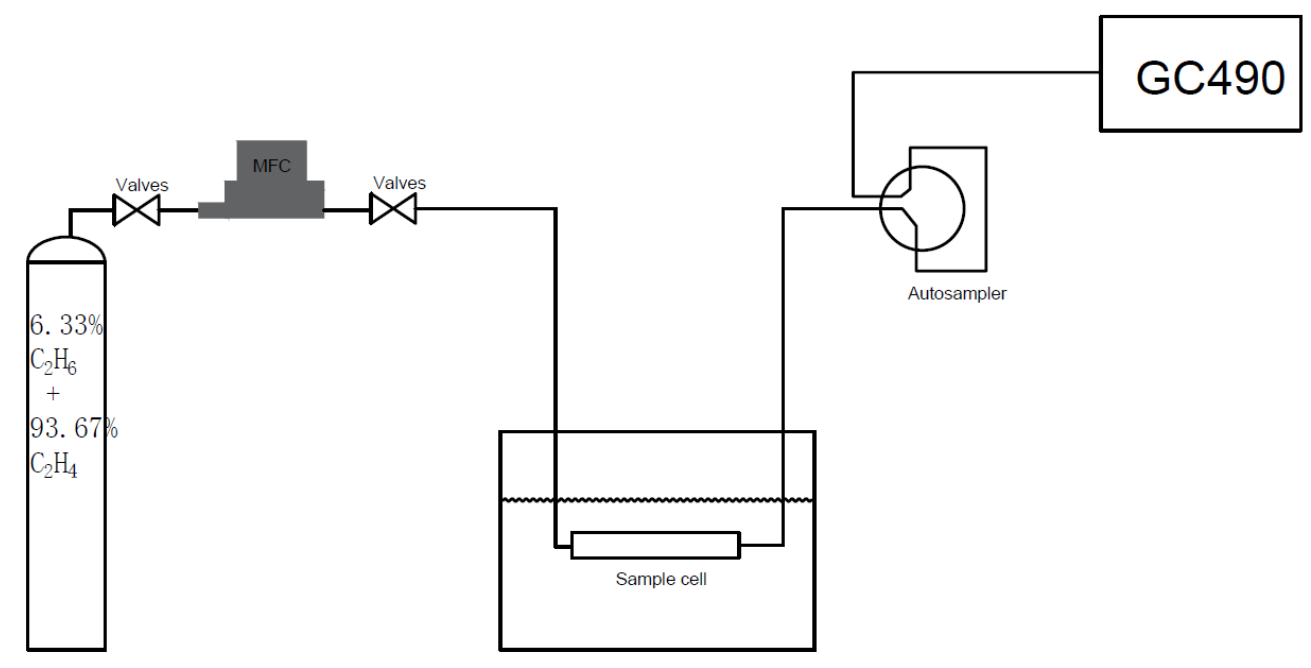

Figure S14. The constructed apparatus for breakthrough experiment. 

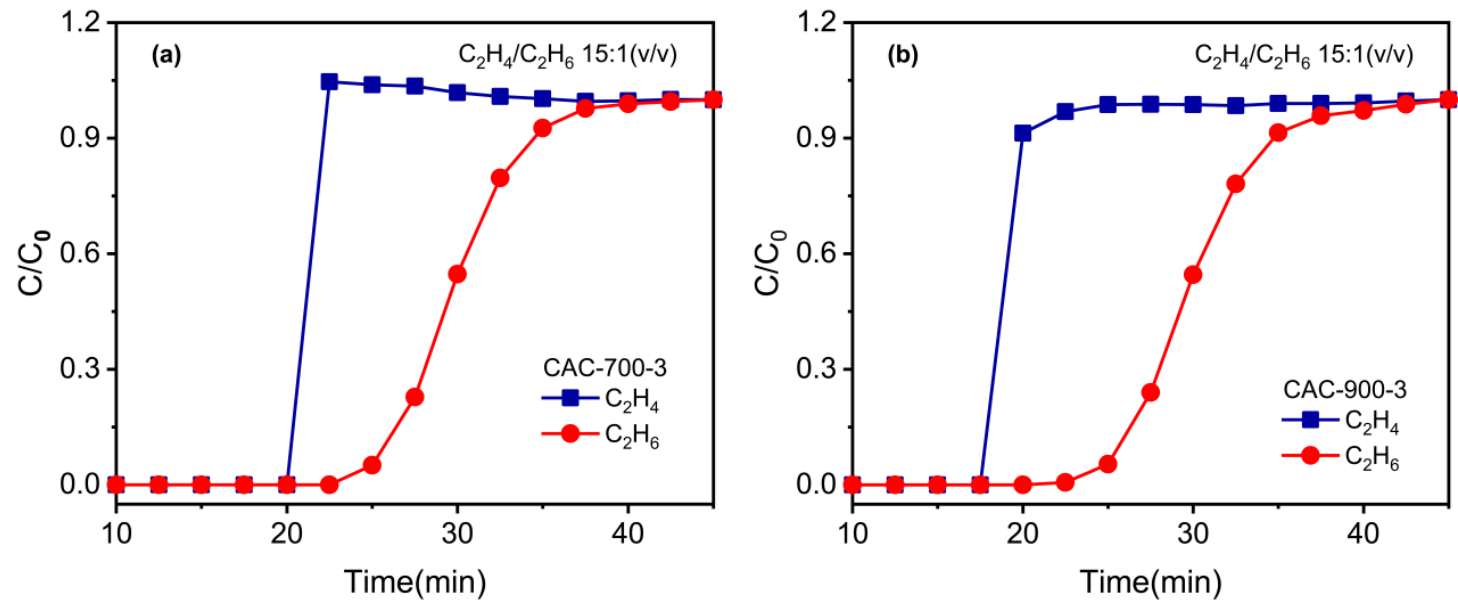

Figure S15. (a) Breakthrough curve s for CAC-700-3 in a fixe $\mathrm{d}$ bed under flow of $\mathrm{C}_{2} \mathrm{H}_{4} / \mathrm{C}_{2} \mathrm{H}_{6}(15 / 1 \mathrm{v} / \mathrm{v})$ gas mixture at 1 bar and $298 \mathrm{~K}$; (b) Breakthrough curve $\mathrm{s}$ for CAC-900-3 in a fixe $d$ bed under flow of $\mathrm{C}_{2} \mathrm{H}_{4} / \mathrm{C}_{2} \mathrm{H}_{6}(15 / 1 \mathrm{v} / \mathrm{v})$ gas mixture at 1 bar and $298 \mathrm{~K}$. 

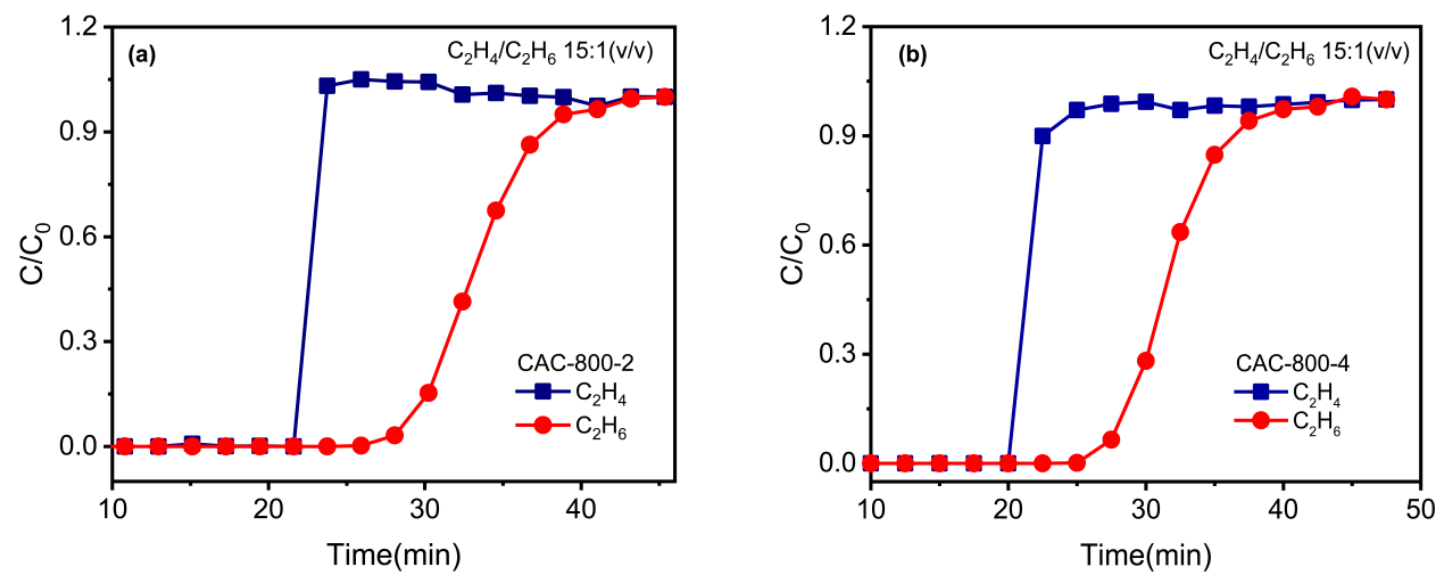

Figure S16. (a) Breakthrough curve s for CAC-800-2 in a fixe d bed under flow of $\mathrm{C}_{2} \mathrm{H}_{4}: \mathrm{C}_{2} \mathrm{H}_{6}(15: 1 \mathrm{v} / \mathrm{v})$ gas mixture at 1 bar and $298 \mathrm{~K}$; (b) Breakthrough curve $\mathrm{s}$ for CAC-800-4 in a fixe $d$ bed under flow of $\mathrm{C}_{2} \mathrm{H}_{4}: \mathrm{C}_{2} \mathrm{H}_{6}(15: 1 \mathrm{v} / \mathrm{v})$ gas mixture at 1 bar and $298 \mathrm{~K}$. 
Table S1. Comparison of physical and chemical properties of $\mathrm{C}_{2} \mathrm{H}_{4}$ and $\mathrm{C}_{2} \mathrm{H}_{6}$

\begin{tabular}{lllll}
\hline Formula & Illustration & $\begin{array}{l}\text { Kinetic } \\
\text { Diameter }\end{array}$ & Boiling Point & $\begin{array}{l}\text { Relative } \\
\text { Volatility }\end{array}$ \\
\hline $\mathrm{C}_{2} \mathrm{H}_{4}$ & $4.163 \AA$ & $-103.9{ }^{0} \mathrm{C}$ & \\
$\mathrm{C}_{2} \mathrm{H}_{6}$ & ${ }^{0}$ & $4.443 \AA$ & $-88.6{ }^{0} \mathrm{C}$ & 1.09 \\
\hline
\end{tabular}

Table S2. Surface carbon and oxygen species of CAC and CAC-X-3 (wt\% of the total carbon or oxygen)

C O

Sample

\begin{tabular}{lllllll} 
& $\mathrm{C}-\mathrm{C}$ & $\mathrm{C}=\mathrm{C}$ & $\mathrm{C}=\mathrm{O}$ & $\mathrm{O}-\mathrm{C}=\mathrm{O}$ & $\mathrm{C}=\mathrm{O}$ & $\mathrm{C}-\mathrm{O}$ \\
\hline Bing & 283.8 & 284.18 & 286.18 & 289.38 & 532.20 & 531.23 \\
energy(eV) & & & & & & \\
CAC & 34.32 & 40.60 & 17.41 & 7.67 & 32.05 & 67.95 \\
CAC-700-3 & 29.94 & 36.50 & 8.74 & 24.22 & 59.14 & 40.86 \\
CAC-800-3 & 35.49 & 37.03 & 12.98 & 14.50 & 65.56 & 34.47 \\
CAC-900-3 & 40.20 & 22.68 & 13.60 & 23.51 & 74.68 & 25.32 \\
\hline
\end{tabular}


Table S3. The fitting parameters of DSLF model and the corresponding correlation coefficients

\begin{tabular}{|c|c|c|c|c|c|c|c|c|}
\hline parameters & & $q_{m, 1}$ & $b_{1}$ & $C$ & $q_{m, 2}$ & $b_{2}$ & $t$ & $R^{2}$ \\
\hline \multirow[t]{2}{*}{ CAC } & $\mathrm{C}_{2} \mathrm{H}_{6}$ & 2.41 & 15.50 & 0.86 & 0.88 & 28.87 & 2.33 & 0.9995 \\
\hline & $\mathrm{C}_{2} \mathrm{H}_{4}$ & 0.96 & 16.58 & 0.88 & 4.62 & 1.15 & 0.76 & 0.9999 \\
\hline \multirow[t]{2}{*}{ CAC-700-3 } & $\mathrm{C}_{2} \mathrm{H}_{6}$ & 8.85 & 1.11 & 0.88 & 1.05 & 25.84 & 0.91 & 0.9999 \\
\hline & $\mathrm{C}_{2} \mathrm{H}_{4}$ & 8.26 & 0.75 & 1.37 & 9.80 & 9.80 & 0.84 & 0.9999 \\
\hline \multirow[t]{2}{*}{ CAC-800-2 } & $\mathrm{C}_{2} \mathrm{H}_{6}$ & 10.05 & 0.99 & 0.96 & 1.34 & 19.28 & 0.91 & 0.9999 \\
\hline & $\mathrm{C}_{2} \mathrm{H}_{4}$ & 1.01 & 16.14 & 0.88 & 9.52 & 0.75 & 0.78 & 0.9999 \\
\hline \multirow[t]{2}{*}{ CAC-800-3 } & $\mathrm{C}_{2} \mathrm{H}_{6}$ & 8.67 & 1.43 & 0.83 & 0.95 & 61.17 & 1.00 & 0.9999 \\
\hline & $\mathrm{C}_{2} \mathrm{H}_{4}$ & 1.01 & 16.14 & 0.88 & 9.52 & 0.75 & 0.78 & 0.9999 \\
\hline \multirow[t]{2}{*}{ CAC-800-4 } & $\mathrm{C}_{2} \mathrm{H}_{6}$ & 0.80 & 10.19 & 51.71 & 0.90 & 1.11 & 0.91 & 0.9999 \\
\hline & $\mathrm{C}_{2} \mathrm{H}_{4}$ & 9.10 & 0.60 & 0.98 & 1.47 & 7.02 & 0.83 & 0.9999 \\
\hline \multirow[t]{2}{*}{ CAC-900-3 } & $\mathrm{C}_{2} \mathrm{H}_{6}$ & 0.93 & 24.50 & 0.92 & 11.01 & 0.85 & 0.89 & 0.9999 \\
\hline & $\mathrm{C}_{2} \mathrm{H}_{4}$ & 8.14 & 0.68 & 1.07 & 1.71 & 7.49 & 0.86 & 0.9999 \\
\hline \multirow[t]{2}{*}{ AAC-800-3 } & $\mathrm{C}_{2} \mathrm{H}_{6}$ & 1.18 & 23.78 & 0.93 & 9.65 & 0.99 & 0.91 & 0.9999 \\
\hline & $\mathrm{C}_{2} \mathrm{H}_{4}$ & 0.54 & 23.73 & 1.02 & 9.96 & 0.70 & 0.79 & 0.9999 \\
\hline \multirow[t]{2}{*}{ WAC-800-3 } & $\mathrm{C}_{2} \mathrm{H}_{6}$ & 8.50 & 1.39 & 0.94 & 1.12 & 38.33 & 1.05 & 0.9999 \\
\hline & $\mathrm{C}_{2} \mathrm{H}_{4}$ & 8.85 & 0.84 & 0.89 & 0.92 & 17.03 & 1.06 & 0.9999 \\
\hline \multirow[t]{2}{*}{ SiFSIX-CAC-800-3 } & $\mathrm{C}_{2} \mathrm{H}_{6}$ & 0.75 & 39.39 & 1.05 & 8.82 & 0.93 & 0.91 & 0.9999 \\
\hline & $\mathrm{C}_{2} \mathrm{H}_{4}$ & 0.65 & 13.87 & 0.93 & 9.66 & 0.56 & 0.84 & 0.9999 \\
\hline \multirow[t]{2}{*}{ HCAC-800-3 } & $\mathrm{C}_{2} \mathrm{H}_{6}$ & 0.59 & 132.14 & 1.28 & 7.22 & 1.23 & 1.28 & 0.9999 \\
\hline & $\mathrm{C}_{2} \mathrm{H}_{4}$ & 7.10 & 0.91 & 0.88 & 1.05 & 15.55 & 0.87 & 0.9999 \\
\hline
\end{tabular}

Table S3 lists the fitting parameters of the DSLF model and the corresponding correlation correlation coefficients $\left(\mathrm{R}^{2}\right)$. The correlation coefficients $\mathrm{R}^{2}$ are up to 0.9995, indicating that the adsorption behaviors of $\mathrm{C}_{2} \mathrm{H}_{6}$ and $\mathrm{C}_{2} \mathrm{H}_{4}$ on ACs can be well described by the DSLF model. 
Table S4. Ethane/ethylene adsorption capacity and IAST selectivity of CAC-X-3 and other ethane-selective adsorbents

\begin{tabular}{|c|c|c|c|c|}
\hline Adsorbent & $\mathrm{Q}_{\text {ethane }}(\mathrm{mmol} / \mathrm{g})$ & $\mathrm{Q}_{\text {ethylene }}(\mathrm{mmol} / \mathrm{g})$ & IAST selectivity & Conditions \\
\hline ZIF-7 & 1.95 & 1.92 & 1.5 & 1 bar and $298 \mathrm{~K}$ \\
\hline ZIF-8 & 2.5 & 1.3 & 1.7 & 1 bar and $293 \mathrm{~K}$ \\
\hline PCN-250 & 5.21 & 4.22 & 1.9 & 1 bar and $298 \mathrm{~K}$ \\
\hline ZIF-69 & 2.2 & 1.8 & 1.7 & 1 bar and $298 \mathrm{~K}$ \\
\hline ZJU-30 & 2.12 & 1.96 & - - & 1 bar and $298 \mathrm{~K}$ \\
\hline MIL-142A & 3.8 & 2.9 & 1.8 & 1 bar and $298 \mathrm{~K}$ \\
\hline IRMOF-8 & 1.6 & 1.1 & 1.8 & $1 \mathrm{bar}$ and $318 \mathrm{~K}$ \\
\hline $\mathrm{Fe}_{2}\left(\mathrm{O}_{2}\right)($ dobdc $)$ & 3.3 & 2.58 & 4.4 & 1 bar and $298 \mathrm{~K}$ \\
\hline $\mathrm{Ni}(\mathrm{bdc})(\mathrm{ted})_{0.5}$ & 5.0 & 3.4 & 1.6 & 1 bar and $298 \mathrm{~K}$ \\
\hline $\mathrm{Cu}(\mathrm{Qc})_{2}$ & 2.19 & 0.92 & 4.1 & 1 bar and $288 \mathrm{~K}$ \\
\hline ANPC-1-800 & 6.84 & 5.71 & 1.53 & 1 bar and $298 \mathrm{~K}$ \\
\hline C-PDA-3 & 6.57 & 5.1 & 1.9 & 1 bar and $298 \mathrm{~K}$ \\
\hline MGA-800-3 & 6.0 & 5.0 & 1.8 & 1 bar and $298 \mathrm{~K}$ \\
\hline 50CPDA@A-AC & 7.12 & 6.25 & 3 & 1 bar and $298 \mathrm{~K}$ \\
\hline Ni1-a & 6.63 & 6.04 & 2 & 1 bar and $298 \mathrm{~K}$ \\
\hline HOF-76a & 2.95 & 1.67 & 2.05 & 1 bar and $296 \mathrm{~K}$ \\
\hline ZJU-HOF-1 & 4.86 & 3.93 & 2.25 & 1 bar and $298 \mathrm{~K}$ \\
\hline ZJU-HOF-10(sc) & 2.19 & 1.88 & 1.9 & 1 bar and $296 \mathrm{~K}$ \\
\hline CAC-700-3 & 5.72 & 4.87 & 1.8 & This work \\
\hline CAC-800-3 & 6.36 & 5.09 & 2.2 & This work \\
\hline CAC-900-3 & 6.02 & 4.86 & 2.6 & This work \\
\hline AAC-800-3 & 5.98 & 4.68 & 2.2 & This work \\
\hline WAC-800-3 & 6.09 & 4.96 & 1.8 & This work \\
\hline SIFSIX-CAC-800-3 & 5.02 & 4.14 & 1.4 & This work \\
\hline HCAC-800-3 & 4.63 & 4.42 & 0.98 & This work \\
\hline
\end{tabular}


Table S5. Cost analysis sheet of CAC-X-Y

\begin{tabular}{ccc}
\hline Material & Cost $/ \mathrm{kg}$ & Yield \\
\hline $\mathrm{CAC}$ & 3 & $\approx 40 \%$ \\
$\mathrm{KOH}$ & 1.5 & \\
\hline
\end{tabular}

